\title{
Training zum Umgang mit Unterrichtsstörungen: Effekte auf die Gesundheit von Lehrkräften
}

\author{
Doris Kunz Heim • Anita Sandmeier • Yves Hänggi • Netkey Safi • \\ Annette Cina
}

Online publiziert: 13. September 2019

(C) Der/die Autor(en) 2019

Zusammenfassung Lehrkräfte sind täglich mit Unterrichtsstörungen in unterschiedlichen Erscheinungsformen und Ausprägungen konfrontiert. Unterrichtsstörungen beeinträchtigen nicht nur das Lernen der Schülerinnen und Schüler, sondern können auch zu einem zentralen Faktor bei der Entstehung von negativen Beanspruchungen bei Lehrpersonen werden. In der vorliegenden Studie wird die Wirkung eines Trainings zum besseren Umgang mit Unterrichtsstörungen (4. bis 6. Schuljahr) auf die wahrgenommene Belastung, die bereichsspezifischen Selbstkognitionen und die negative Beanspruchung von Lehrkräften untersucht. Im Rahmen einer längsschnittlichen Quasi-Experimentalstudie mit einem Pretest und drei Posttests $\left(N_{\text {Interventionsgruppe }}=65, N_{\text {Kontrollgruppe }}=53\right)$ zeigte sich anhand varianzanalytischer Auswertungen, dass in der Interventionsgruppe die Belastung und die negative Beanspruchung durch Unterrichtsstörungen abnehmen und sich die bereichsspezifischen Selbstkognitionen verbessern.

Schlüsselwörter Beanspruchung · Lehrergesundheit · Selbstkognition · Trainingswirkung · Unterrichtsstörungen

Prof. Dr. D. Kunz Heim $(\bowtie) \cdot$ N. Safi $(\bowtie)$

Fachhochschule Nordwestschweiz, Pädagogische Hochschule, Bahnhofstrasse 5, 5210 Windisch, Schweiz

E-Mail: doris.kunz@fhnw.ch; netkey.safi@fhnw.ch

Prof. Dr. A. Sandmeier $(\varangle)$

Pädagogische Hochschule Schwyz, Zaystraße 42, 6410 Goldau, Schweiz

E-Mail: anita.sandmeier@phsz.ch

Dr. Y. Hänggi $(\varangle) \cdot$ Dr. A. Cina $(\varangle)$

Institut für Familienforschung und -beratung, Universität Fribourg, Rue P.A.

Faucigny 2, 1700 Fribourg, Schweiz

E-Mail: yves.haenggi@gmx.ch; annette.cina@unifr.ch 


\section{Training in classroom management: Effects on teacher well-being}

Abstract Every day, teachers face all kinds of disruptive student behavior in the classroom. Disruptions in the classroom interfere with student learning and can be a key driver of negative strain and adverse health outcomes for teachers. This study analyses the effects of a training in classroom management (4th to 6th grade) on relevant factors of teacher occupational well-being. A quasi-experimental longitudinal design was applied in a four-wave panel-study including a treatment group $(N=65)$ and a control group $(N=53)$. Analysis of variance revealed a decrease in demands and strain in the treatment group and an increase of teachers' positive self-cognition with regard to handling disruptive behavior.

Keywords Disruptive pupil behavior - Negative strain - Self-cognition · Teacher well-being · Training effectiveness

\section{Einleitung}

Lehrerinnen und Lehrer sind im Beruf hoch zufrieden, aber auch stark belastet (Bieri 2006; Schult et al. 2014). Überbeanspruchungen von Lehrkräften stehen im Zusammenhang mit negativen gesundheitlichen Folgen wie Erschöpfung und Depression und einer geringeren Unterrichtsqualität (Klusmann et al. 2006). Schülerinnen und Schüler nehmen die negative Beanspruchung ihrer Lehrpersonen wahr und weisen geringere Motivation und Leistungen auf (Klusmann et al. 2008). Als zentraler Faktor, der zu negativen Beanspruchungen von Lehrpersonen führt, sind Unterrichtsstörungen in ihren verschiedenen Erscheinungsformen identifiziert worden (Hakanen et al. 2006; Clunies-Ross et al. 2008). Der Umgang mit Unterrichtsstörungen ist demnach eine Kompetenz, die nicht nur grundlegend ist für gelingenden Unterricht, sondern auch ein Faktor, der im Zusammenhang mit der Gesundheit von Lehrpersonen steht. Ophardt et al. (2017) stellen fest, dass die gezielte Aus- und Weiterentwicklung von Kompetenzen im Klassenmanagement und somit im Umgang mit Unterrichtsstörungen in der Aus- und Weiterbildung der Lehrkräfte bisher noch wenig weit gediehen ist. Klusmann und Philipp (2014) weisen auf erste Erfolge von Lehrertrainings hin, die zur Förderung von Ressourcen (z. B. verbesserte Kompetenz) beitragen und dadurch zu einer Verringerung der gesundheitsgefährdenden Folgen. Bisher sind jedoch nur wenige Studien bekannt, welche die Wirkung von lehrerspezifischen Trainings zum Umgang mit Unterrichtsstörungen auf die gesundheitlichen Folgen bei Lehrkräften untersuchen. Deren Ergebnisse sind uneinheitlich (Dicke et al. 2015; Neuenschwander und Benini 2016).

In der vorgestellten, vom Schweizerischen Nationalfonds und vom Bundesamt für Gesundheit geförderten Studie wird die Wirkung eines neu entwickelten Lehrertrainings zum besseren Umgang mit Unterrichtsstörungen untersucht. Die Fragestellung lautet, ob das Training zu einer Reduktion der Belastung der Lehrpersonen durch Unterrichtsstörungen führt. Zudem werden die Effekte des Trainings auf die bereichsspezifischen Selbstkognitionen und die negative Beanspruchung untersucht. 


\section{Theoretischer Hintergrund}

\subsection{Belastung und Beanspruchung}

Transaktionale Erklärungsmodelle für die Entstehung von Beanspruchung verstehen Belastungen als von außen auf den Menschen einwirkende Einflüsse (Rudow 2000; Ulich und Wülser 2004). Die daraus entstehende Beanspruchung meint eine Erlebnisqualität, welche von der individuellen Bewertung der Bedeutung der Situation und von den zur Verfügung stehenden Ressourcen beeinflusst wird (Lazarus und Launier 1981; Ulich und Wülser 2004). Bei den Ressourcen kann unterschieden werden zwischen unterstützenden Faktoren im Kontext und funktionalen Eigenschaften und Kompetenzen der Person, die zur Bewältigung der Belastungen beitragen.

Ein spezifisches Erklärungsmodell für die Entstehung von berufsbedingter Beanspruchung ist das Job Demands-Resources (JD-R) Model, welches ebenfalls von der Relation zwischen Belastungen und Ressourcen ausgeht, jedoch von der individuellen Bewertung abstrahiert. Im Modell sagen berufsbedingte Belastungen negative Beanspruchungsreaktionen wie Stress und Erschöpfung voraus. Ressourcen haben eine lindernde Wirkung auf die negativen und darüber hinaus eine fördernde Wirkung auf positive Beanspruchungsreaktionen wie z. B. die Selbstkognitionen in Form von kognitiver Aktivierung. Das JD-R-Modell konnte während der letzten zehn Jahre im Rahmen von zahlreichen Studien bestätigt werden und zählt aktuell zu einem der führenden Erklärungsmodelle für berufsbedingte Beanspruchung (Bakker und Demerouti 2007; Schaufeli und Taris 2014). Auf dieses Modell stützt sich das heuristische Modell der vorliegenden Studie (Abb. 1). Es wird postuliert, dass durch das Training die Ressourcen der Lehrpersonen für den Umgang mit Unterrichtsstörungen erhöht werden. Dadurch nehmen die wahrgenommene Belastung und die negativen Beanspruchungsreaktionen (Stress und Erschöpfung) ab und die positiven Beanspruchungsreaktionen (kognitive Aktivierung) nehmen zu.

\subsection{Die Bedeutung von Unterrichtsstörungen für das Beanspruchungserleben von Lehrpersonen}

Störendes Schülerverhalten beeinträchtigt nicht nur das Lernen der Kinder in einer Lerngruppe, sondern es ist auch eine der im Zusammenhang mit negativen Beanspruchungsreaktionen bei Lehrpersonen besonders häufig genannten Belastungen (Burke et al. 1996; Brouwers und Tomic 2000; Hakanen et al. 2006; Clunies-Ross et al. 2008; Aloe et al. 2014). In einer älteren Studie von Mykletun (1984) zeigte sich, dass unter neun für Lehrpersonen relevanten Belastungsfaktoren störendes Schülerverhalten nach dem Faktor Arbeitsüberlastung den zweitstärksten Zusam-

Abb. 1 Heuristisches Modell der Studie (nach Bakker und Demerouti 2007; Schaufeli und Taris 2014)

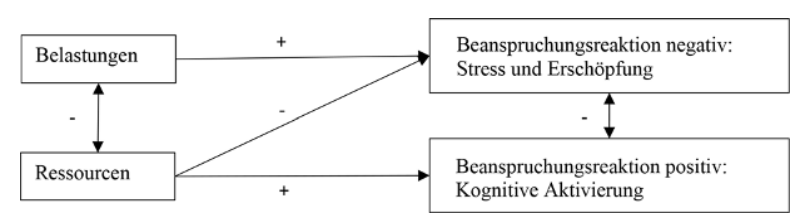


menhang zu gesundheitlichen Störungen mit depressiver Symptomatik aufweist. Kokkinos (2007) identifizierte elf für Lehrpersonen relevante Belastungsfaktoren und fand regressionsanalytisch den stärksten Zusammenhang zwischen störendem Schülerverhalten und Burn-out. Strukturgleichungsmodelle zeigten, dass Störungen im Unterricht nach dem Faktor Arbeitsüberlastung und Konflikten mit Eltern den drittstärksten Effekt auf das Erschöpfungserleben von Lehrpersonen hatten (Baeriswyl et al. 2014).

\subsection{Die Bedeutung der Selbstkognition für das Beanspruchungserleben von Lehrpersonen}

In bisherigen Studien hat sich gezeigt, dass zwei Komponenten der Selbstkognition das Beanspruchungserleben von Lehrpersonen günstig beeinflussen: Die Selbstwirksamkeit und das Kompetenzselbstkonzept (Pajares und Schunk 2001).

Selbstwirksamkeit beschreibt die Überzeugung des Individuums, dass es in wechselnden und auch schwierigen Situationen seine verschiedenen Fähigkeiten erfolgreich (neu) organisieren kann, um eine bestimmte Anforderung zu bewältigen (Bandura 1997). Selbstwirksamkeit kann dabei allgemein (Jerusalem und Schwarzer 1999) oder mehrdimensional und domänenspezifisch verstanden werden, z.B. mit den Dimensionen der Selbstwirksamkeit in Bezug auf den Unterricht, auf die Klassenführung oder auf das Schülerengagement (Schwarzer und Schmitz 1999; Tschannen-Moran und Woolfolk-Hoy 2001; Skaalvik und Skaalvik 2007). Zahlreiche Studien machen deutlich, dass die Selbstwirksamkeit eine abschwächende Wirkung auf negative Beanspruchungsreaktionen von Lehrpersonen hat (Brouwers und Tomic 2000; Evers et al. 2002; Skaalvik und Skaalvik 2007; Fussangel et al. 2010; Aloe et al. 2014; Dicke et al. 2014; Fussangel und Dizinger 2014; Schwarzer und Warner 2014).

Im Rahmen der vorliegenden Studie steht die Selbstwirksamkeit bezüglich der Klassenführung und des Umgangs mit Unterrichtsstörungen im Zentrum. Aloe et al. (2014) führten eine Metaanalyse von 16 Studien zum Zusammenhang von Selbstwirksamkeit für die Klassenführung (SWKF) und den drei Teildimensionen von Burn-out durch. Den stärksten (positiven) Zusammenhang fanden sie zwischen der SWKF und der wahrgenommenen eigenen Leistungsfähigkeit, gefolgt vom negativen Zusammenhang zwischen SWKF und Depersonalisation sowie zwischen SWKF und emotionaler Erschöpfung, wobei diese Befunde nichts über die Kausalität aussagen. Eine Studie von Brouwers und Tomic (2000) mit zwei Messzeitpunkten weist darauf hin, dass das Ausmaß der emotionalen Erschöpfung die SWKF negativ beeinflusst und nicht umgekehrt. Dicke et al. (2014, 2018) fanden im Rahmen von Studien mit Referendaren in Deutschland, dass die SWKF einen abschwächenden Effekt auf die emotionale Erschöpfung hat, dass sich dieser Effekt auch im Längsschnitt zeigt und bei jenen Lehrkräften besonders stark ist, die eine niedrige oder mittlere Selbstwirksamkeit aufweisen.

Die Bedeutung des Kompetenzselbstkonzeptes für das Beanspruchungserleben von Lehrpersonen wurde erst wenig untersucht. Das Kompetenzselbstkonzept weist Ähnlichkeiten mit dem Konstrukt der Selbstwirksamkeit auf. Es ist jedoch im Zeitverlauf stabiler (Skaalvik und Skaalvik 2017) und hat eine andere Grundausrichtung, 
indem es einen bewertenden Anteil in Bezug auf eigene bereichsspezifische Fähigkeiten hat (Pajares und Schunk 2001). Während die Selbstwirksamkeitsüberzeugung sich auf das Vertrauen bezieht, das jemand in den erfolgreichen Einsatz seiner Fähigkeiten hat, ist das Selbstkonzept ein Element der Beschreibung des Selbst, dessen bewertende Komponente die individuelle Einschätzung der eigenen Fähigkeiten in einem bestimmten Bereich umfasst. Die entsprechende Bewertung beruht auf einem Vergleich mit Fähigkeiten von anderen Personen (Pajares und Schunk 2001). In zwei Studien wurden positive Effekte des Kompetenzselbstkonzeptes auf das Arbeitsengagement und die Arbeitszufriedenheit sowie ein negativer Effekt auf die Ausprägung von Burn-out-Symptomen von Lehrerinnen und Lehrern gefunden (Kunz Heim et al. 2014a; Skaalvik und Skaalvik 2017).

\subsection{Wirkungen von Trainingsprogrammen zum Umgang mit Unterrichtsstörungen}

Trainings zum Umgang mit Unterrichtsstörungen sind im Kontext des Klassenmanagements angesiedelt. Das Klassenmanagement zielt erstens darauf ab, eine geordnete Lernumgebung zu gewährleisten, in der es den Schülerinnen und Schülern möglich ist, sich auf Lernprozesse zu konzentrieren, und in der zweitens auch die soziale und moralische Entwicklung der Lernenden gefördert wird (Evertson und Weinstein 2006; Schönbächler 2008; Babad 2009). Ein diesbezüglich im englischsprachigen Raum weitverbreitetes Training ist das Incredible Years Teacher Classroom Management Program (Hutchings et al. 2013).

In zahlreichen Studien sind Effekte solcher Trainings auf das Schüler- und Lehrerverhalten nachgewiesen worden, die meisten Studien mit Bezug auf die Primarstufe (Stage und Quiroz 1997; Babyak et al. 2000; Chafouleas et al. 2012; Neuenschwander und Benini 2016). Stage und Quiroz (1997) fanden in ihrer Metaanalyse von 99 Studien, dass es möglich ist, das störende Verhalten von Schülerinnen und Schülern in öffentlichen Schulen durch Trainings deutlich zu reduzieren.

Effekte von Trainings zum Klassenmanagement auf das Beanspruchungserleben und die Selbstkognition von Lehrpersonen wurden bisher erst in wenigen Studien untersucht. Die Untersuchung eines Klassenführungstrainings für Referendare ergab positive Effekte auf die Schlafqualität der Lehrpersonen und mildernde auf deren emotionale Erschöpfung (Dicke et al. 2015). Bei einem Training zur Förderung von Kindern mit Aufmerksamkeitsdefizit-Hyperaktivitäts-Störungen (ADHS) wurden positive Effekte auf das Kompetenzerleben der Lehrkräfte gefunden, aber keine generellen Effekte auf deren Belastungswahrnehmung (Neuenschwander und Benini 2016). In der Untersuchung von Hagen et al. (1998) wurden positive Effekte von stellvertretenden positiven Erfahrungen (Betrachtung von Videoaufnahmen von gutem Klassenmanagement) auf die Selbstwirksamkeit der Lehrpersonen gefunden. Nach einem Training zum Klassenmanagement, das spezifisch auf die Erhöhung der Selbstwirksamkeit der Lehrpersonen ausgerichtet war, wurde beobachtet, dass die personale Selbstwirksamkeit in Bezug auf den Unterricht und die spezifische personale Selbstwirksamkeit in Bezug auf den Umgang mit schwierigem Schülerverhalten sich wie erwartet positiv veränderten (Gaudreau et al. 2013). 


\section{Das Training}

Die vorliegende Interventionsstudie untersucht die Wirkung des von den Mitautoren an der Universität Freiburg (Schweiz) neu entwickelten Trainings Unterrichtsstörungen sicher begegnen (USIB) auf die Selbstkognitionen und das Beanspruchungserleben von ausgebildeten Lehrpersonen. Die Evaluation basiert auf einem quasiexperimentellen Prä-Post-Design mit einer Interventions- und Kontrollgruppe sowie vier Messzeitpunkten innerhalb von 12 Monaten.

\subsection{Ziel und Inhalt des Trainings}

Durch das neu entwickelte Training sollen die Kompetenzen im Umgang mit schwierigem Schülerverhalten gestärkt und Handlungsmöglichkeiten aufgezeigt werden, wie eine positive Beziehung zu den Schülerinnen und Schülern aufgebaut, erwünschtes Verhalten von Lernenden gestärkt und in anspruchsvollen Situationen adäquat und konsequent reagiert werden kann. Ziel der Intervention ist dadurch gleichzeitig, ein positives Klassenklima, eine Stärkung der Selbstständigkeit der Lernenden sowie eine Abnahme von Störverhalten der Schülerinnen und Schüler zu bewirken. Das Training will außerdem die Lehrpersonen anregen, die Hintergründe des problematischen Verhaltens des Kindes zu analysieren, dieses zu beobachten und zu überlegen, welches Verhalten das Kind noch erwerben oder verstärken muss.

Das umfassende Ziel des Trainings in Bezug auf das heuristische Modell der Studie ist (vgl. Abb. 1), die Ressourcen der Lehrpersonen in Form von funktionalen Kompetenzen im Umgang mit Unterrichtsstörungen zu erhöhen.

\subsection{Grundlagen und Struktur des Trainings}

Das Lehrpersonentraining stützt sich auf ähnlich aufgebaute Trainings für Eltern im Rahmen der Erziehungsberatung. Es ist modular aufgebaut und umfasst vier Halbtagesmodule à 3,5 h, welche aufeinander aufbauen und im Wochenabstand während des Schuljahres stattfinden. Der beabsichtigte Aufbau der Ressourcen der Lehrpersonen für den Umgang mit Unterrichtsstörungen erfolgt aufgrund von vier Elementen: Die Lehrpersonen werden erstens in den Modulen über die theoretischen Hintergründe ausgebildet (Wissensvermittlung), sie werden zweitens geschult, sich über Ursachen von Unterrichtsstörungen in der eigenen Klasse und ihren Umgang damit bewusst zu werden (Verhaltensbeobachtung und Selbstreflexion), sie entwickeln drittens alternative Verhaltensweisen auf der Basis der Verhaltensanalyse sowie der vorgestellten Verhaltensformen (Verhaltensänderung a) und üben viertens zwischen den Modulen die ausgewählten Strategien und Umgangsformen mit dem Verhalten der Schülerinnen und Schüler (Übung). Im Rahmen des ergänzenden Telefoncoachings können die Lehrpersonen Unterstützung bei der Umsetzung in Anspruch nehmen (Verhaltensänderung b). Die Inhalte der Module lauten:

Modul 1: Beobachtung von Ursachen und Bedingungen des störenden Verhaltens. Ausgehend von verhaltenspsychologischen Überlegungen (Reinecker 2005) wird das Bewusstsein der Lehrpersonen dazu angeregt, mögliche Ursachen des störenden Schülerverhaltens zu vertiefen und die Bedingungen $\mathrm{zu}$ beobachten, unter denen es 
aufrechterhalten wird, damit diese so verändert werden können, dass sich auch das Verhalten der Kinder ändert (Patterson 1982; Reid et al. 2002). Ergänzend werden den Lehrpersonen die Wirkmechanismen von Verhaltensanpassungen aufgezeigt.

Modul 2: Aufbau von positivem Verhalten der Schülerinnen und Schüler. Die Inhalte dieses Moduls basieren auf der instrumentellen, sozialen und kognitiven Lerntheorie (Bandura 1977; Patterson 1982; Pianta et al. 2012) und umfassen die Bereiche Aufbau einer positiven Beziehung zwischen Schülern und Lehrpersonen als Basis, Verstärken der funktionalen Fertigkeiten der Schülerinnen und Schüler sowie das Vermitteln von sozial angemessenen Verhaltensweisen.

Modul 3: Umgang mit Problemverhalten, basiert auf denselben Grundlagen wie Modul 2. Es werden mögliche Strategien dargestellt und erarbeitet, wie auf aktuell auftretendes Problemverhalten während des Unterrichts reagiert werden kann, wie z. B. ein auf das Problemverhalten fokussiertes Aufstellen von Regeln, direktes Ansprechen beim Übertreten von Regeln und mögliche adäquate logische Konsequenzen auf das Problemverhalten.

Modul 4: Umgang mit Risikosituationen und Führen von Elterngesprächen. In diesem Modul werden erstens die bisher erworbenen Kenntnisse und Fertigkeiten vertieft. Zweitens werden Strategien zum Umgang mit schwierigen Risikosituationen erarbeitet und drittens geht es um die Verbesserung der Gesprächsführung mit Eltern, um diese bestmöglich in den Lernprozess der Kinder einzubinden.

\section{Methode}

\subsection{Forschungsfragen und Hypothesen}

Die übergeordnete Forschungsfrage lautet, ob das Training einen Einfluss hat auf die im heuristischen Modell (vgl. Abb. 1) dargestellten relevanten Faktoren. Überprüft wird erstens die Wirkung des Trainings auf die wahrgenommene Belastung. Dabei wird angenommen, dass die durch das Training erworbenen neuen Ressourcen zu einer Verringerung der wahrgenommenen Belastung durch Unterrichtsstörungen führen. Es wird erwartet, dass die Teilnehmenden nach dem Training weniger Unterrichtsstörungen wahrnehmen.

Zweitens wird die Wirkung des Trainings auf die wahrgenommenen positiven Beanspruchungsreaktionen überprüft. Dabei wird angenommen, dass sich das höhere $\mathrm{Maß}$ an funktionalen Ressourcen positiv auf die Selbstkognitionen auswirkt. Es wird erwartet, dass nach dem Training mehr Selbstwirksamkeit und ein höheres Maß an Kompetenzselbstkonzept wahrgenommen werden.

Drittens wird die Wirkung des Trainings auf die wahrgenommenen negativen Beanspruchungsreaktionen überprüft. Es wird erwartet, dass sich erstens das höhere $\mathrm{Ma} ß$ an funktionalen Ressourcen und zweitens das geringere Maß an wahrgenommenen Unterrichtsstörungen günstig auswirken. Es wird erwartet, dass die Teilnehmenden nach dem Training weniger Stress durch störendes Schülerverhalten wahrnehmen. 
Die Hypothesen basieren auf den soeben beschriebenen angenommenen Wirkmechanismen und lauten: Das Training hat

- einen förderlichen Effekt auf das wahrgenommene Ausmaß von Unterrichtsstörungen, das heißt, dass nach dem Training weniger Unterrichtsstörungen wahrgenommen werden (H1: weniger Belastung),

- einen positiven Effekt auf die Ausprägung der Selbstkognitionen im Umgang mit Unterrichtsstörungen, das heißt, dass nach dem Training mehr Selbstwirksamkeit und ein höheres Maß an Kompetenzselbstkonzept wahrgenommen werden als vorher (H2: mehr positive Beanspruchungsreaktionen),

- einen förderlichen Effekt auf die Ausprägung der wahrgenommenen Beanspruchung durch Unterrichtsstörungen, das heißt, dass nach dem Training weniger Stress durch störendes Schülerverhalten wahrgenommen wird (H3: weniger negative Beanspruchungsreaktionen).

\subsection{Trainingsorganisation und Durchführungskonstanz}

Das Training für die Interventionsgruppe fand in der unterrichtsfreien Zeit der Lehrpersonen statt und wurde in sieben Kursen in fünf Deutschschweizer Städten durchgeführt (Aarau, Bern, St. Gallen, Winterthur, Zürich). Die Trainingsgruppen umfassten zwischen sechs und vierzehn Lehrpersonen.

Um alle Trainings der Interventionsgruppe möglichst gleichzeitig durchführen zu können, konnten acht Trainer/innen (7 weiblich, 1 männlich) gewonnen werden, die bereits als Trainer/innen im Rahmen der Erziehungsberatung in Familien ausgebildet waren und beruflich mit Schulen arbeiteten.

Um die Durchführungskonstanz des neu für die Lehrpersonen entwickelten Trainings zu gewährleisten, wurde dieses in einem Manual ausführlich verschriftlicht (Cina et al. 2012). Alle Trainer/innen wurden im Rahmen einer eintägigen Weiterbildung in die Besonderheiten des Lehrertrainings eingeführt.

\subsection{Design der Studie}

Die Studie ist aufgebaut als längsschnittliche Quasi-Experimentalstudie mit einem Pretest und drei Posttests, einer Interventions- und einer Warte-Kontrollgruppe. Der Vortest (t0) erfolgte vor der Intervention im September 2012, der erste Posttest (t1) im Januar 2013, der zweite Posttest (t2) im Mai 2013 und der dritte Posttest (t3) ein Jahr nach dem Pretest im Oktober 2013. Die Lehrpersonen der Kontrollgruppen füllten die Fragebogen zu denselben Zeitpunkten aus, nahmen aber erst nach den Messungen im Oktober 2013 an der Intervention teil.

\subsection{Stichprobe}

Die Teilnehmenden wurden in allen Deutschschweizer Kantonen über Publikationen der Bildungsverwaltungen und der Lehrerverbände sowie mit einem Schreiben an alle Schulleitungen der Primarstufe rekrutiert. Das Ziel, 140 Lehrpersonen für die freiwillige Teilnahme am Training und den Befragungen zu gewinnen, konnte bis 
Tab. 1 Sozioprofessionelle Merkmale der Interventions- und der Kontrollgruppe

\begin{tabular}{lllll}
\hline Merkmale & $\begin{array}{l}\text { Gesamte Stich- } \\
\text { probe } \\
(n=129)\end{array}$ & $\begin{array}{l}\text { Interventionsgruppe } \\
(n=71)\end{array}$ & $\begin{array}{l}\text { Kontrollgruppe } \\
(n=58)\end{array}$ & Vergleichstest \\
\hline Anteil Frauen $(\%)$ & $80,6 \%$ & $85,9 \%$ & $74,1 \%$ & $C h i^{2}(1)=2,834 ;$ \\
$(N)$ & $(104)$ & $(61)$ & $(43)$ & $p=0,092$ \\
Berufserfahrung $M$ & 3,43 & 3,45 & 3,40 & $t=0,266$ \\
$(\mathrm{SD})$ & $(1,14)$ & $(1,17)$ & $(1,13)$ & $p=0,101$ \\
Anzahl Lektionen & 21,82 & 20,45 & 23,89 & $t=-3,358$ \\
pro Woche $M(S D)$ & $(6,16)$ & $(6,43)$ & $(5,11)$ & $p=0,001$ \\
Anzahl Kinder & 18,59 & 18,69 & 18,48 & $t=0,266$ \\
pro Klasse $M(S D)$ & $(4,28)$ & $(4,33)$ & 3,38 & $p=0,790$ \\
Anz. schwieriger & 3,52 & 3,63 & $(1,94)$ & $t=0,770$ \\
Schüler $M(S D)$ & $(1,83)$ & $(1,75)$ & 2,90 & $p=0,443$ \\
Störverhalten* der & 2,72 & 2,58 & $(1,05)$ & $t=-1,770$ \\
Klasse $M(S D)$ & $(1,1)$ & $(0,96)$ & & $p=0,079$ \\
\hline
\end{tabular}

$n$ Anzahl Personen, $C h i^{2}$ Chi-Quadrat-Test ( $\mathrm{df}=$ Freiheitsgrade), $p$ Irrtumswahrscheinlichkeit, $t=\mathrm{T}$-Test, $M$ Mittelwert, $S D$ Standardabweichung, *Störverhalten der Klasse in fünfstufiger Skalierung $(1=$ sehr oft; $5=$ sehr selten)

zwei Monate vor Beginn des Trainings nicht erreicht werden. Aus diesem Grund wurden alle bis zu diesem Zeitpunkt angemeldeten Lehrpersonen der Interventionsgruppe zugeteilt und die Kontrollgruppe nachrekrutiert. Bei dieser Nachrekrutierung für die Kontrollgruppe wurde die Teilnahme am Training fakultativ angeboten. Die Evaluation des Trainings basiert demnach auf einer nichtrandomisierten Stichprobe.

Zum Messzeitpunkt t0 konnten 129 Personen befragt werden, 71 in der Interventions- und 58 in der Kontrollgruppe. Interventions- und Kontrollgruppe wurden hinsichtlich der Unterschiede bezüglich sozioprofessioneller Merkmale untersucht (Tab. 1). Zu diesem Zweck wurden $t$-Tests und $C h i^{2}$-Tests durchgeführt. Die beiden Gruppen unterscheiden sich in einem der sechs überprüften Merkmale: Die Kontrollgruppe unterrichtet durchschnittlich mehr Lektionen pro Woche $(M=23,89)$ als die Interventionsgruppe $(M=20,45)$.

Im Verlauf der Studie fielen sechs Personen in der Interventionsgruppe (Dropout-Rate $=8,4 \%$ ) und fünf Personen in der Kontrollgruppe (Dropout-Rate $=8,6 \%$ ) aus, so dass schließlich 118 Primarlehrpersonen (Klassen 4 bis 6) zu allen Messzeitpunkten befragt werden konnten (Interventionsgruppe $n=65$, Kontrollgruppe $n=53$ ). Diese Stichprobe variiert aufgrund von spezifischen Ausfällen je nach Variable minimal in Bezug auf die vier abhängigen Variablen.

\subsection{Instrumente}

Das wahrgenommene Ausmaß an Unterrichtsstörungen wurde mit der Skala „Aktives Stören“ (Schönbächler 2005) erhoben, die in sechs Items das Vorhandensein von verschiedenen Unterrichtsstörungen erfasst (Bsp: Die Schülerinnen und Schüler schwatzen manchmal während Erklärungsphasen). Die Antwortvorgaben der fünfstufigen Likert-Skala sind (1) trifft gar nicht zu bis (5) trifft völlig zu $(\alpha=0,74)$. Was die positiven Beanspruchungsreaktionen in Form der Selbstkognitionen betrifft, wur- 
de a) die Selbstwirksamkeit und b) das Kompetenzselbstkonzept erhoben. Für die Erhebung der Selbstwirksamkeit bezüglich des Umgangs mit Unterrichtsstörungen wurde die von Schönbächler (2005) übersetzte Ohio State Teacher Efficacy Scale verwendet (Tschannen-Moran und Woolfolk-Hoy 2001). Sie umfasst acht Items (Bsp. Können Sie störendes Verhalten im Klassenzimmer kontrollieren?) mit einer fünfstufigen Skala $(1)=$ gar nicht bis $(5)=$ in großem Maß $(\alpha=0,85)$. Das Kompetenzselbst konzept im Umgang mit Schülerinnen und Schülern wurde mit vier Items aus der Skala von Kunz Heim und Rindlisbacher (2009) erhoben (Bsp. Wie kompetent fühlen Sie sich in Bezug auf die Fähigkeit: Problemverhalten von Schülern/Schülerinnen im Unterricht auffangen können?) und vier Antwortstufen (1)=weniger kompetent bis (4) sehr kompetent $(\alpha=0,77)$. Die wahrgenommene negative Beanspruchung durch Unterrichtsstörungen wurde mit der deutschen Übersetzung (Baeriswyl et al. 2014) der Skala „Störendes Schülerverhalten“ (Hakanen et al. 2006) erhoben (Bsp: Wie häufig fühlen Sie sich in Ihrer Lehrtätigkeit durch einzelne Schüler/innen gestresst, die sich ständig schlecht benehmen?). Die Antwortvorgaben der neun Items weisen fünf Stufen auf (1) nie oder fast nie bis (5) sehr oft $(\alpha=0,85)$.

\subsection{Datenanalyse}

Um sicherzustellen, dass die zentralen Konstrukte in der Interventions- und der Kontrollgruppe das Gleiche bedeuten, wurde in Voranalysen die Messinvarianz der vier abhängigen Variablen entlang der Empfehlungen von Van de Schoot et al. (2012) mit dem Paket lavaan in R geprüft (Rosseel 2012). Alle weiteren Analysen wurden mit SPSS 24 mit manifesten Skalen vorgenommen. In Voranalysen wurde außerdem mittels $t$-Tests überprüft, ob die Interventionsgruppe und die Kontrollgruppe sich in der Prämessung signifikant voneinander unterscheiden.

Die Frage, ob sich die abhängigen Variablen über die Zeit, abhängig von der Gruppenzugehörigkeit, verändern, wurde mit einer mehrfaktoriellen Varianzanalyse mit Messwiederholung (Bortz und Döring 2006) mit den Faktoren Gruppe (Interventionsgruppe/Kontrollgruppe) und Messzeitpunkt und deren Interaktion geprüft. Der Haupteffekt des Faktors Gruppe sagt etwas darüber aus, ob sich die beiden Gruppen aggregiert über die Messzeitpunkte unterscheiden. Der Haupteffekt des Faktors Zeit erlaubt eine Aussage darüber, ob sich die fragliche abhängige Variable über die Zeit verändert, unabhängig von der Intervention. Der Interaktionseffekt von Gruppe*Zeit erlaubt die Aussage, ob sich die abhängige Variable, abhängig von der Intervention, über die Zeit verändert. Neben der Frage, ob es einen signifikanten Effekt der Intervention in der erwünschten Richtung gibt, interessiert die Stärke dieses Effektes. Aus diesem Grund werden die Effektstärken der Veränderungen zwischen den vier Messzeitpunkten für die beiden Gruppen berechnet. Die Voraussetzungen für die Varianzanalysen wurden teilweise verletzt, insbesondere die Varianzhomogenität (Levene-Test) und die Sphärizität (Mauchly-Test). Verletzung der Normalverteilung und der Varianzhomogenität ist bei genügend großen Stichproben und gleicher Zellenbesetzung weniger problematisch (vgl. Bortz und Döring 2006). Im Beitrag werden die Ergebnisse des multivariaten Signifikanztests berichtet, welcher robuster ist gegenüber Verletzungen der Voraussetzungen. Bei Verletzung der Sphärizität wurde die Greenhouse-Geisser-Korrektur angewendet. 


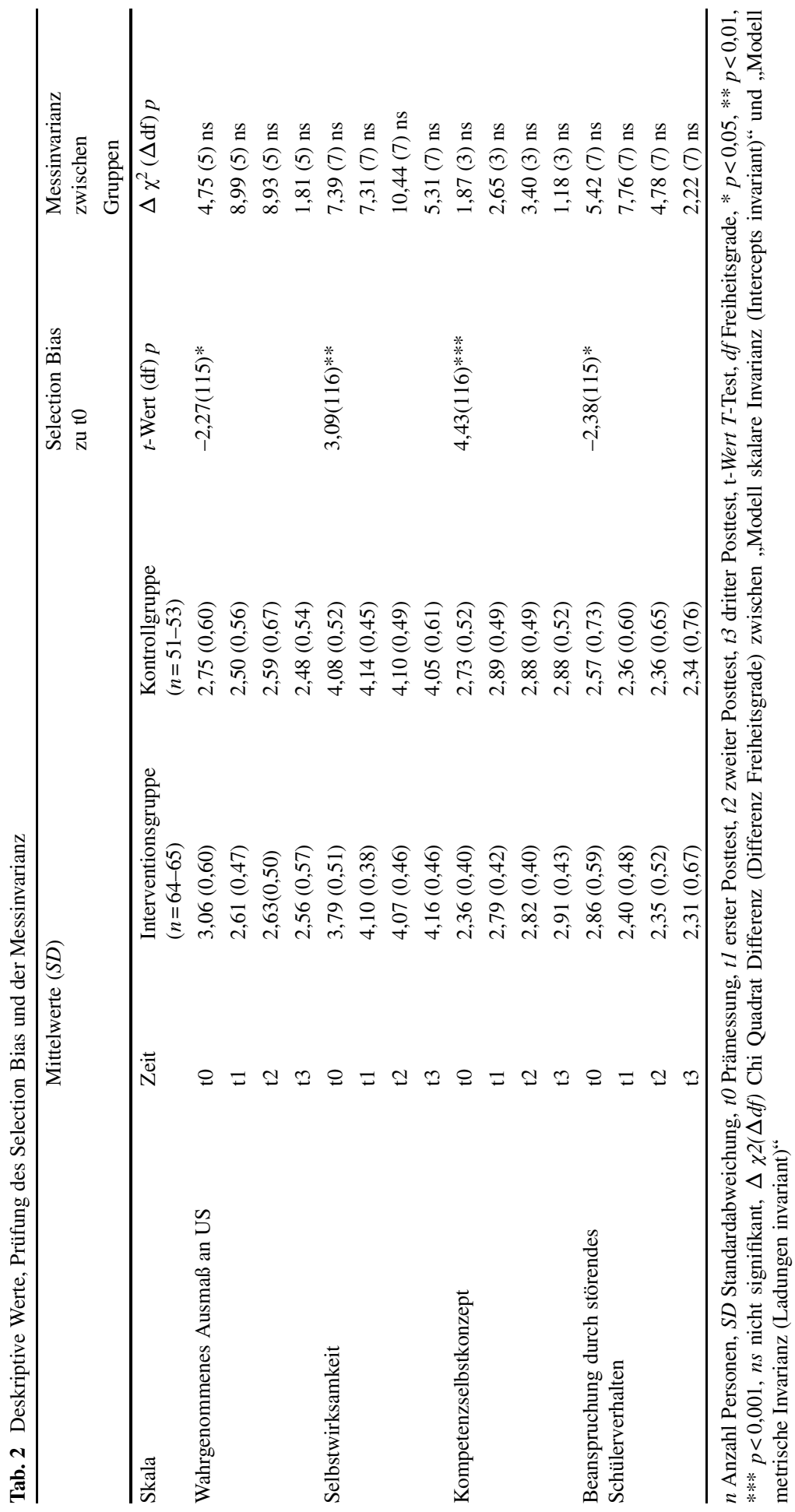


Um die praktische Relevanz der Veränderungen in der Interventionsgruppe einschätzen zu können, wurden die Effektstärken $\left(e t a^{2}\right)$ der Veränderungen für die Interventionsgruppe berechnet, indem der Wert der Prämessung (t0) mit den Postmessungen (t1, t2, t3) verglichen wird. Die Prämessung erfolgte, wie erwähnt, einen Monat vor der Intervention. Sie wird mit der ersten Postmessung t1 (rund drei Monate nach der Intervention), der zweiten Postmessung t2 (rund sechs Monate nach der Intervention) und dem Follow-up nach 12 Monaten (t3) verglichen.

\subsection{Voranalysen}

Messinvarianz: Die konfirmatorische Faktoranalyse ergab, dass für alle Konstrukte zu allen vier Messzeitpunkten skalare Messinvarianz zwischen den beiden Gruppen besteht (vgl. Tab. 2). Die Ladungen und Intercepts der einzelnen Items können über die beiden Gruppen zu jedem Messzeitpunkt gleichgesetzt werden, ohne dass sich der Fit des Messmodells signifikant verschlechtert (Van de Schoot et al. 2012). Das ist die Grundlage dafür, dass man die manifesten Mittelwerte der beiden Gruppen valide miteinander vergleichen kann.

Selection Bias Aus Tab. 2 können sowohl die Mittelwerte und Standardabweichungen der vier Skalen für die vier Messzeitpunkte (t0 bis t3) entnommen werden als auch die Resultate der Gruppenvergleiche (unabhängige $t$-Tests, zweiseitig).

Im ersten Messzeitpunkt (Prämessung) liegen signifikante Unterschiede zwischen der Interventions- und der Kontrollgruppe vor: Die Interventionsgruppe nimmt ein höheres Ausmaß an Unterrichtsstörungen wahr $(t(115)=-2,27, p<0,05)$, hat eine geringere Selbstwirksamkeit $(t(116)=3,09, p<0,01)$, ein negativeres Kompetenzselbstkonzept $(t(116)=4,43, p<0,000)$ und ist stärker beansprucht durch störendes Schülerverhalten $(t(115)=-2,38, p<0,05)$ als die Kontrollgruppe. Es handelt sich hier möglicherweise um einen Selection-Bias (Lehr 2014), der sich aufgrund der weiter oben beschriebenen Besonderheiten der Rekrutierung ergeben hat. Diejenigen Lehrpersonen, die sich in der ersten Rekrutierungswelle freiwillig für die Studie und das Training angemeldet hatten und die der Interventionsgruppe zugeordnet wurden, fühlen sich durch den Umgang mit Unterrichtsstörungen deutlich stärker belastet und beansprucht und weisen deutlich ungünstigere Selbstkognitionen auf als die Lehrpersonen der Kontrollgruppe, die bei der zweiten Welle rekrutiert wurden. Wie erwähnt wurden Letztere lediglich für die Messungen angefragt und die Teilnahme am Training war freiwillig ${ }^{1}$.

\section{Ergebnisse}

\subsection{Resultate der ANOVAs mit Messwiederholung}

Im Folgenden werden die Resultate zum Einfluss der Intervention auf die verschiedenen abhängigen Variablen vorgestellt. Das partielle Eta-Quadrat $\left(\eta^{2}\right)$ zeigt als Maß

\footnotetext{
1 Von der Kontrollgruppe $(N=55)$ nahmen 12 Personen nach Abschluss der Studie am Training teil.
} 
Tab. 3 Effektstärken der Veränderung für die Interventionsgruppe (t-Tests)

\begin{tabular}{lllllll}
\hline Skala & $\mathrm{t} 0$ bis $\mathrm{t} 1$ & \multicolumn{3}{c}{$\mathrm{t} 0 \mathrm{bis} \mathrm{t} 2$} & \multicolumn{2}{c}{$\mathrm{t} 0$ bis $\mathrm{t} 3$} \\
& $p$ & eta $^{2}$ & $p$ & eta $^{2}$ & $p$ & eta $^{2}$ \\
\hline Wahrgenommenes Ausmaß an US & 0,013 & 0,053 & 0,004 & 0,068 & 0,047 & 0,034 \\
Selbstwirksamkeit & 0,002 & 0,083 & 0,003 & 0,073 & 0,000 & 0,139 \\
Kompetenzselbstkonzept & 0,001 & 0,097 & 0,001 & 0,095 & 0,000 & 0,154 \\
Beanspruchung durch störendes & 0,032 & 0,040 & 0,006 & 0,063 & 0,044 & 0,035
\end{tabular}

Schülerverhalten

$t 0$ Prämessung, $t 1$ erster Posttest, $t 2$ zweiter Posttest, $t 3$ dritter Posttest, $p$ Irrtumswahrscheinlichkeit, eta $^{2}$ Eta Quadrat: 0,01 klein, 0,06 mittel, 0,14 gross (Cohen 1988)

der Effektstärke an, wie viel Variation durch den Faktor erklärt wird. In Tab. 3 finden sich die Effektstärken $\left(e t a^{2}\right)$ der Veränderungen für die Interventionsgruppe und in den Abb. 2 und 3 werden exemplarisch die Mittelwerte von zwei abhängigen Variablen zu den vier Messzeitpunkten grafisch dargestellt.

\subsection{Einfluss der Intervention auf das wahrgenommene Ausmaß an Unterrichtsstörungen}

Beim wahrgenommenen Ausmaß an Unterrichtsstörungen liegen sowohl ein Haupteffekt über die Zeit $\left(F(2,71,306,02)=24,45, p=0,000, \eta^{2}=0,178\right)$ als auch eine signifikante Interaktion zwischen Zeit und Gruppe vor $(F(2,71,306,02)=2,918$, $\left.p=0,040, \eta^{2}=0,025\right)$. In Tab. 2 ist ersichtlich, dass das wahrgenommene Ausmaß an Unterrichtsstörungen in der Interventionsgruppe zwischen t0 und $\mathrm{t} 1$, also direkt nach der Intervention, abnimmt und sich der Einschätzung in der Kontrollgruppe annähert.

Betrachtet man die Effektstärken der Veränderungen für die Interventionsgruppe (Tab. 3) in der Dimension des wahrgenommenen Ausmaßes an Unterrichtsstörungen (US), ist der Effekt zwischen der Prämessung t0 und der zweiten Postmessung t2 sechs Monate nach der Intervention am höchsten und liegt im mittleren Bereich $\left(e t a^{2}=0,068\right)$. Im Vergleich zwischen der Prämessung mit der ersten sowie der dritten Postmessung $\mathrm{t} 1$ und $\mathrm{t} 3$ zeigen sich ebenfalls signifikante Effekte, die jedoch geringer sind $\left(e t a^{2}=0,053 ; e t a^{2}=0,034\right)$.

\subsection{Einfluss der Intervention auf die Selbstkognitionen (wahrgenommene positive Beanspruchungsreaktionen)}

Wenn man die Veränderung der Selbstwirksamkeit der beiden Gruppen analysiert, zeigen sich ein signifikanter Haupteffekt über die Zeit $(F(2,77,321,58)=7,821$, $\left.p=0,000, \eta^{2}=0,063\right)$ und eine signifikante Interaktion zwischen Zeit und Gruppe $\left(F(2,77,321,58)=8,044, p=0,000, \eta^{2}=0,065\right)$. In Tab. 2 ist ersichtlich, dass die Selbstwirksamkeit in der Interventionsgruppe zwischen t0 und t 1 deutlich steigt, um sich danach zu stabilisieren, während in der Kontrollgruppe die Selbstwirksamkeit über alle Messzeitpunkte hinweg relativ stabil bleibt.

Beim domänenspezifischen Kompetenzselbstkonzept (Abb. 2) sind sowohl der Haupteffekt über die Zeit $\left(F(2,78,319,84)=28,591, p=0,000, \eta^{2}=0,199\right)$ als auch 
4,00

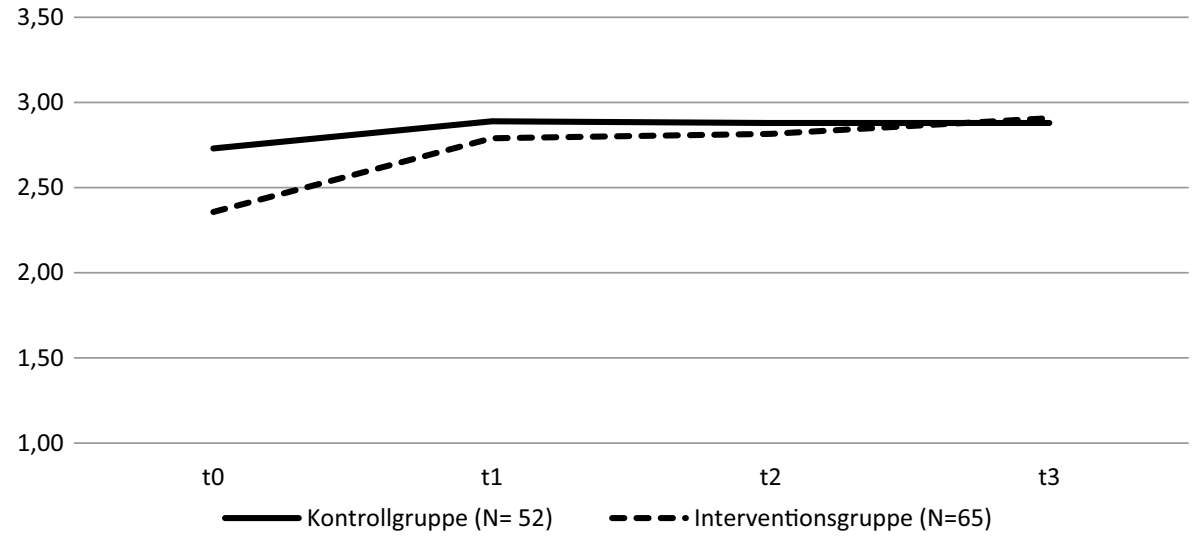

Abb. 2 Kompetenzselbstkonzept bezüglich Unterrichtsstörungen nach Gruppe über die Zeit

\subsection{0}

3.50

3.00

2.50

2.00

1.50

1.00

to

t1

--- Kontrollgruppe $(\mathrm{N}=51)$

t2

t3

Abb. 3 Beanspruchung durch störendes Schülerverhalten nach Gruppe über die Zeit

die Interaktion zwischen Zeit und Gruppe signifikant $(F(2,78,319,84)=9,632$, $\left.p=0,000, \eta^{2}=0,077\right)$. In der Abbildung ist ersichtlich, dass sich in der Interventionsgruppe das Kompetenzselbstkonzept von t0 bis $\mathrm{t} 3$ stetig verbessert mit dem größten Anstieg zwischen t0 und t1.

Betrachtet man die Effektstärken der Veränderungen für die Interventionsgruppe (Tab. 3), zeigen sich bei der Selbstwirksamkeit zwischen t0 und der ersten und zweiten Postmessung t1 und 2 Effekte im mittleren Bereich $\left(\right.$ et $a^{2}=0,083 ;$ et $\left.a^{2}=0,073\right)$ und zwischen t0 und $\mathrm{t} 3$ im hohen Bereich $\left(e t a^{2}=0,139\right)$. Eine ähnliche Ausprägung zeigt die Veränderung des Kompetenzselbstkonzepts. Auch hier liegen die Effekte im Vergleich zwischen der Prämessung und den Postmessungen $\mathrm{t} 1$ und $\mathrm{t} 2$ im mittle- 
ren Bereich $\left(e t a^{2}=0,097 ; e t a^{2}=0,095\right)$ und im Vergleich zwischen der Prämessung und der Postmessung $\mathrm{t} 3$ im hohen Bereich $\left(e t a^{2}=0,154\right)$.

\subsection{Einfluss der Intervention auf den wahrgenommenen Stress durch störendes Schülerverhalten (wahrgenommene negative Beanspruchungsreaktion)}

Für den wahrgenommenen Stress durch störendes Schülerverhalten (Abb. 3) findet sich ein signifikanter Effekt über die Zeit $(F(2,31,261,06)=15,413, p=0,000$, $\left.\eta^{2}=0,120\right)$ und für die Interaktion zwischen Zeit und Gruppe $(F(2,31,261,06)=3,439$, $\left.p=0,027, \eta^{2}=0,030\right)$. Die Verläufe zwischen den beiden Gruppen sind ähnlich, wobei in der Interventionsgruppe zwischen t0 und t1 eine stärkere Verringerung der wahrgenommenen Beanspruchung zu beobachten ist.

Die Effektstärken der Veränderungen bei der Interventionsgruppe bezüglich der wahrgenommenen negativen Beanspruchung durch störendes Schülerverhalten (Tab. 3) zeigen zwischen $\mathrm{t} 0$ und $\mathrm{t} 1$ und zwischen $\mathrm{t} 0$ und $\mathrm{t} 3$ Effekte im niedrigen Bereich $\left(e t a^{2}=0,040 ; e t a^{2}=0,035\right)$ und Effekte im mittleren Bereich zwischen t0 und $\mathrm{t} 2\left(e t a^{2}=0,063\right)$.

\section{Diskussion}

Ziel der vorliegenden Studie war es, die Wirkung eines neu entwickelten Trainings zum Umgang mit Unterrichtsstörungen auf die wahrgenommene Belastung und das Beanspruchungserleben von Lehrpersonen zu untersuchen. Das Training zielte darauf ab, die Ressourcen der Lehrkräfte für den Umgang mit Unterrichtstörungen zu verbessern. Dadurch, so wurde aufgrund des heuristischen Modells (Abb. 1) postuliert, nimmt das wahrgenommene Ausmaß an Unterrichtsstörungen ab (Hypothese 1, weniger Belastung). Weiter wurde angenommen, dass die verstärkten Ressourcen der Lehrpersonen eine positive Wirkung auf deren Selbstkognitionen haben (Hypothese 2, mehr positive Beanspruchungsreaktionen). Schließlich wurde erwartet, dass die verstärkten Ressourcen und die wahrgenommene Abnahme der Unterrichtsstörungen zu einer wahrgenommenen Abnahme des Stresses durch störendes Schülerverhalten führen (Hypothese 3, weniger negative Beanspruchungsreaktionen).

Die Hypothesen wurden im Rahmen eines quasi-experimentellen Prä-Post-Designs mit einer Interventions- und einer Kontrollgruppe überprüft. Die Rekrutierung der Stichprobe gestaltete sich als schwierig, weshalb die Zuordnung zu den Gruppen nicht randomisiert verlaufen konnte. Die Stichprobe weist einen Selektionsbias auf, der sich darin zeigt, dass die Teilnehmenden, die sich auf die erste Ausschreibung angemeldet hatten und der Interventionsgruppe zugeteilt wurden, bei der Prämessung signifikant höhere Werte im Bereich der Belastung und der negativen Beanspruchung und signifikant niedrigere Werte im Bereich der Selbstkognitionen aufwiesen als die Teilnehmenden der Kontrollgruppe. Die gefundenen Ergebnisse müssen aus diesem Grund vorsichtig interpretiert werden und können nur als erster Befund verstanden werden, der in weiteren Studien validiert werden muss.

Die Analysen zeigen, dass das wahrgenommene Ausmaß an Unterrichtsstörungen in der Interventionsgruppe erwartungsgemäß abnimmt, und zwar am stärksten 
zwischen der Prämessung und der Messung sechs Monate später. Die Mittelwerte bleiben auf einem niedrigeren Niveau auch in der Postmessung ein Jahr nach der Intervention, d.h., es kann diesbezüglich von einer langfristigen Wirkung des Trainings ausgegangen werden (Hypothese 1). Eine deutliche Erhöhung der Werte wurde erwartungsgemäß für das bereichsspezifische Kompetenzselbstkonzept und die Selbstwirksamkeit registriert: In der Interventionsgruppe zeigte sich, im Gegensatz zur Kontrollgruppe, eine deutliche Verbesserung in diesen beiden Dimensionen der Selbstkognition (Hypothese 2). Auch Hypothese 3 konnte beibehalten werden. In der Interventionsgruppe nahm die Beanspruchung durch störendes Schülerverhalten $\mathrm{ab}$.

Die Fragestellung des Beitrags, ob durch eine Weiterbildung zur Verbesserung des Umgangs mit Unterrichtsstörungen eine Belastungs- und Beanspruchungsreduktion sowie eine Verbesserung der Selbstkognition bei Lehrkräften erzielt werden kann, lässt sich aufgrund der vorliegenden Analysen mit einem vorsichtigen „Ja, aber“ beantworten. Die erwarteten Veränderungen konnten zwar beobachtet werden. Fragen wirft jedoch der Umstand auf, wonach sowohl bei der Interventions- als auch bei der Kontrollgruppe Effekte über die Zeit vorliegen, d.h., dass die Werte der wahrgenommenen Belastung und Beanspruchung nach dem Training niedriger und diejenigen der Selbstwirksamkeit und des Kompetenzselbstkonzepts höher ausfallen. Es ist möglich, dass die Teilnehmenden der Kontrollgruppe, die sich auf die zweite Ausschreibung der Studie gemeldet hatten, zu jenem Zeitpunkt in einer zeitlich begrenzten Belastungssituation befunden haben, z. B. bei der Übernahme einer „schwierigen“ Klasse. Die beobachteten Veränderungen lassen jedoch verhaltenen Optimismus aufkommen. Die Ergebnisse zeigen tendenziell, dass das Training einerseits präventiv wirkt, indem es dazu beiträgt, eine der bedeutendsten Quellen von Belastung und Beanspruchungen von Lehrpersonen zu verringern, und andererseits gesundheitsfördernd, indem es zur Verbesserung der Selbstkognitionen beiträgt.

Nebst der bereits erwähnten Einschränkung durch die fehlende Randomisierung und dem damit verbundenen Selektionsbias muss auch auf die offenen Fragen bezüglich der Validität der in der Studie verwendeten Selbsteinschätzungen hingewiesen werden. Was die wahrgenommene Belastung betrifft, fanden Stage und Quiroz (1997) in der erwähnten Metaanalyse deutlich größere Trainingseffekte in Studien, bei denen die abhängigen Variablen durch Beobachtung des Schülerverhaltens erhoben wurden als bei solchen mit Einschätzungen durch die Lehrpersonen selbst, was die Autoren als niedrigere Sensitivität der Lehrer-Ratings für Veränderungen durch die Trainings deuten. In Bezug auf die Messung der Beanspruchungsreaktionen müsste mit Ergänzungen durch alternative Erhebungsverfahren ${ }^{2}$ die Validität der Selbsteinschätzungen kontrolliert werden.

Ungeklärt ist zudem die Frage nach dem Response Shift, einer möglichen Bedeutungsänderung des gemessenen Konstruktes bei den Befragten zwischen Vor- und Posttests. Piwowar und Thiel (2014) fanden, dass sich zu dessen Überprüfung ein latenter Ansatz der Messinvarianzprüfung von separaten Komponenten des Mess-

\footnotetext{
2 In Bezug auf die negative Beanspruchung zum Beispiel mit Hilfe eines Sensor-Armbandes zur Messung physiologischer Stressreaktionen (Papastefanou 2013).
} 
modells über die Zeit eignet, ein Verfahren, das jedoch eine größere Stichprobe als die in der Studie vorhandene erfordert.

Die Stichprobengröße der Interventionsgruppe $(n=65)$ führte auch dazu, dass das eingangs vorgestellte heuristische Modell (vgl. Abb. 1) nicht als Gesamtmodell im Rahmen eines Strukturgleichungsmodells überprüft werden konnte. Weil das Job Demands-Resources Model, auf das sich die Analysen beziehen, wie erwähnt mehrfach und mit Stichproben aus den unterschiedlichsten Professionen in zahlreichen europäischen und außereuropäischen Ländern bestätigt werden konnte (Schaufeli und Taris 2014) und zugleich kein Mediationsmodell darstellt, weist es eine sehr geeignete theoretische Passung für die vorgenommene varianzanalytische Auswertung auf.

Um die Frage nach der Wirkung des Trainings schlüssig beantworten zu können, müssen die gefunden Tendenzen im Rahmen eines randomisierten Kontrollgruppendesigns bestätigt werden, bei dem kein Selektions-Bias vorliegt. Die zusätzliche Messung der abhängigen Variablen durch alternative Verfahren wird dazu beitragen, die gefundenen Resultate zusätzlich zu validieren. Es braucht somit weitere Studien, welche die Wirkung von Trainings zum besseren Umgang mit Unterrichtsstörungen untersuchen, damit Trainings (weiter)entwickelt werden können, die evidenzbasiert eine wirkungsvolle Verbesserung der Ressourcen der Lehrpersonen im Umgang mit Unterrichtsstörungen ermöglichen.

Eingangs wurde erwähnt, dass erst wenige Studien vorliegen, die zeigen, dass Lehrertrainings Ressourcen erhöhen und so zur Gesundheitsförderung beitragen können. Die vorliegende Studie liefert einen Hinweis darauf, dass durch solche Trainings die Ressourcen der Teilnehmenden bezüglich des Umgangs mit schwierigem Schülerverhalten verbessert werden können und sich dadurch die Selbstkognitionen der Lehrpersonen erhöhen und deren Belastung und Beanspruchung verringern. Wenn berücksichtigt wird, dass rund ein Drittel der Lehrkräfte stark von negativen Beanspruchungsreaktionen wie Stress und Erschöpfung betroffen sind (van Dick und Wagner 2001; Kunz Heim et al. 2014b), ist es von zentraler Bedeutung, dass solche Trainings zur gezielten Förderung von Kompetenzen im Bereich der Klassenführung in der Aus- und Weiterbildung von Lehrerinnen und Lehrern einen festen Platz erhalten.

Open Access Dieser Artikel wird unter der Creative Commons Namensnennung 4.0 International Lizenz (http://creativecommons.org/licenses/by/4.0/deed.de) veröffentlicht, welche die Nutzung, Vervielfältigung, Bearbeitung, Verbreitung und Wiedergabe in jeglichem Medium und Format erlaubt, sofern Sie den/die ursprünglichen Autor(en) und die Quelle ordnungsgemäß nennen, einen Link zur Creative Commons Lizenz beifügen und angeben, ob Änderungen vorgenommen wurden.

\section{Literatur}

Aloe, A.M., Amo, L.C., \& Shanahan, M.E. (2014). Classroom management self-efficacy and burnout: a multivariate meta-analysis. Educational Psychology Review, 26(1), 101-126.

Babad, E. (2009). The social psychology of the classroom. New York: Routledge.

Babyak, A.E., Luze, G.J., \& Kamps, D. M. (2000). The good student game: behavior management for diverse classrooms. Intervention in School and Clinic, 35(4), 216-223.

Baeriswyl, S., Krause, A., \& Kunz Heim, D. (2014). Arbeitsbelastungen, Selbstgefährdung und Gesundheit bei Lehrpersonen - eine Erweiterung des Job Demands-Resources Modells. Empirische Pädagogik, 28(2), 128-146. 
Bakker, A.B., \& Demerouti, E. (2007). The job demands-resources model: state of the art. Journal of Managerial Psychology, 22(3), 309-328.

Bandura, A. (1977). Self-efficacy: toward a unifying theory of behavioral change. Psychological Review, 84(2), 191-215.

Bandura, A. (1997). Self-efficacy. The exercise of control. New York: W. H. Freeman and Company.

Bieri, T. (2006). Lehrpersonen: Hoch belastet und trotzdem zufrieden? Bern: Haupt.

Bortz, J., \& Döring, N. (2006). Forschungsmethoden und Evaluation für Human- und Sozialwissenschaftler (Limitierte Sonderausgabe). Heidelberg: Springer.

Brouwers, A., \& Tomic, W. (2000). A longitudinal study of teacher burnout and perceived self-efficacy in classroom management. Teaching and Teacher Education, 16, 239-253.

Burke, R.J., Greenglass, E.R., \& Schwarzer, R. (1996). Predicting teacher burnout over time: Effects of work stress, social support, and self-doubts on burnout and its consequences. Anxiety, Stress \& Coping: An International Journal, 9(3), 261-275.

Chafouleas, S. M., Hagemoser Sanetti, L. M., Jaffery, R., \& Fallon, L. M. (2012). An evaluation of a classwide intervention package involving self-management and a group contingency on classroom behavior of middle school students. Journal of Behavioral Education, 21(1), 34-57.

Cina, A., Hänggi, Y., \& Kurz, S. (2012). Unterrichtsstörungen sicher begegnen. Ein verhaltenspsychologisches Training für Lehrpersonen zum Umgang mit schwierigem Schülerverhalten. Freiburg: Universität Freiburg.

Clunies-Ross, P., Little, E., \& Kienhuis, M. (2008). Self-reported and actual use of proactive and reactive classroom management strategies and their relationship with teacher stress and student behaviour. Educational Psychology, 28(6), 693-710.

Cohen, J. (1988). Statistical power analysis for the behavioral sciences. New York: L. Erlbaum Associates.

Dicke, T., Elling, J., Schmeck, A., \& Leutner, D. (2015). Reducing reality shock: the effects of classroom management skills training on beginning teachers. Teaching and Teacher Education, 48, 1-12.

Dicke, T., Parker, P. D., Marsh, H., Kunter, M., Schmeck, A., \& Leutner, D. (2014). Self-efficacy in classroom management, classroom disturbances, and emotional exhaustion: A moderated mediation analysis of teacher candidates. Journal of Educational Psychology, 106(2), 569-583.

Dicke, T., Stebner, F., Linninger, C., Kunter, M., \& Leutner, D. (2018). A longitudinal study of teachers' occupational well-being: applying the job demands-resources model. Journal of Occupational Health Psychology, 23(2), 262-277.

Evers, W. J., Brouwers, A., \& Tomic, W. (2002). Burnout and self-efficacy: a study on teachers' beliefs when implementing an innovative educational system in the Netherlands. British Journal of Educational Psychology, 72, 227-243.

Evertson, C. M., \& Weinstein, C. S. (2006). Classroom management as a field of inquiry. In C. M. Evertson (Hrsg.), Handbook of classroom management. Research, practice and contemporary issues (S. 3-15). Mahwah: Erlbaum.

Fussangel, K., \& Dizinger, V. (2014). The challenge of change? The development of all-day schools and its implications for teacher stress. Journal for Educational Research Online, 6(3), 115-133.

Fussangel, K., Dizinger, V., Boehm-Kasper, O., \& Graesel, C. (2010). Kooperation, Belastung und Beanspruchung von Lehrkräften an Halb- und Ganztagsschulen. Cooperation, stress, and strain of teachers at half-day and all-day schools. Unterrichtswissenschaft, 38(1), 51-67.

Gaudreau, N., Royer, E., Frenette, E., Beaumont, C., \& Flanagan, T. (2013). Classroom behaviour management: The effects of in-service training on elementary teachers' self-efficacy beliefs. McGill Journal of Education, 48(2), 359-382.

Hagen, K. M., Gutkin, T.B., Wilson, C.P., \& Oats, R. G. (1998). Using vicarious experience and verbal persuasion to enhance self-efficacy in pre-service teachers: "Priming the pump" for consultation. School Psychology Quarterly, 13(2), 169-178.

Hakanen, J. J., Bakker, A. B., \& Schaufeli, W. B. (2006). Burnout and work engagement among teachers. Journal of School Psychology, 43, 495-513.

Hutchings, J., Martin-Forbes, P., Daley, D., \& Williams, M. E. (2013). A randomized controlled trial of the impact of a teacher classroom management program on the classroom behavior of children with and without behavior problems. Journal of School Psychology, 51(5), 571-585.

Jerusalem, M., \& Schwarzer, R. (Hrsg.). (1999). Skala zur allgemeinen Selbstwirksamkeitserwartung. Skalen zur Erfassung von Lehrer- und Schülermerkmalen. Dokumentation der psychometrischen Verfahren im Rahmen der Wissenschaftlichen Begleitung des Modellversuchs Selbstwirksame Schulen. Berlin: Freie Universität Berlin.

Klusmann, U., \& Philipp, A. (2014). Belastung und Beanspruchung im Lehrerberuf: Zum Stand der empirischen Forschung. In E. Terhart, H. Bennewitz \& M. Rothland (Hrsg.), Handbuch der Forschung zum Lehrerberuf (2. Aufl., S. 1014-1022). Münster: Waxmann. 
Klusmann, U., Kunter, M., Trautwein, U., \& Baumert, J. (2006). Lehrerbelastung und Unterrichtsqualität aus der Perspektive von Lehrenden und Lernenden. Zeitschrift für Pädagogische Psychologie, 20(3), $161-173$.

Klusmann, U., Kunter, M., Trautwein, U., Lüdtke, O., \& Baumert, J. (2008). Teachers' occupational wellbeing and quality of instruction: The important role of self-regulatory patterns. Journal of Educational Psychology, 100(3), 702-715.

Kokkinos, C. M. (2007). Job stressors, personality and burnout in primary school teachers. British Journal of Educational Psychology, 77, 229-243.

Kunz Heim, D., \& Rindlisbacher, S. (2009). Die Verbreitung des Weiterlernens von Lehrpersonen: Effekte der Praxisgemeinschaft und des Kompetenzselbstkonzeptes. Schweizerische Zeitschrift für Bildungswissenschaften, 31(3), 497-518.

Kunz Heim, D., Sandmeier, A., \& Krause, A. (2014a). Effekte von arbeitsbedingten und personalen Ressourcen auf das Arbeitsengagement und das Engagement für die Schulentwicklung bei Lehrpersonen. Empirische Pädagogik, 28(2), 147-170.

Kunz Heim, D., Sandmeier, A., \& Krause, A. (2014b). Negative Beanspruchungsfolgen bei Schweizer Lehrpersonen. Beiträge zur Lehrerinnen- und Lehrerbildung, 32(2), 280-295.

Lazarus, R.S., \& Launier, R. (1981). Stressbezogene Transaktionen zwischen Person und Umwelt. In R. Nitsch (Hrsg.), Stress. Theorien, Untersuchungen, Maßnahmen (S. 213-259). Bern: Huber.

Lehr, D. (2014). Belastung und Beanspruchung im Lehrerberuf - Präventions- und Interventionsansätze in der personenbezogenen Forschung. In I. E. Terhart, H. Bennewitz \& M. Rothland (Hrsg.), Handbuch der Forschung zum Lehrerberuf (2. Aufl., S. 968-986). Münster: Waxmann.

Mykletun, R. J. (1984). Teacher stress: Perceived and objective sources, and quality of life. Scandinavian Journal of Educational Research, 28(1), 17-45.

Neuenschwander, M. P., \& Benini, S. (2016). FOKUS. Förderung von Kindern mit Verhaltensauffälligkeiten und Unaufmerksamkeit im Unterricht. Schlussbericht für das Bundesamt für Gesundheit. Solothurn: Fachhochschule Nordwestschweiz, Pädagogische Hochschule, Institut Forschung und Entwicklung, Zentrum Lernen und Sozialisation. http://www.fhnw.ch/ph/zls/fokus. Zugegriffen: 7. September 2016.

Ophardt, D., Piwowar, V., \& Thiel, F. (2017). Kompetenzen des Klassenmanagements (KODEK). In C. Gräsel \& K. Trempler (Hrsg.), Entwicklung von Professionalität pädagogischen Personals: Interdisziplinäre Betrachtungen, Befunde und Perspektiven (S. 133-152). Wiesbaden: Springer.

Pajares, F., \& Schunk, D.H. (2001). Self-beliefs and school success: self-efficacy, self-concept, and school achievement. In R. J. Riding \& S. G. Rayner (Hrsg.), Self Perception. International Perspectives on Individual Differences (Bd. 2, S. 239-265). Westport: Ablex Publishing.

Papastefanou, G. (2013). xperimentelle Validierung eines Sensor-Armbandes zur mobilen Messung physiologischer Stress-Reaktionen. GESIS-Technical Reports, 2013/07. Mannheim: GESIS - LeibnizInstitut für Sozialwissenschaften.

Patterson, G. R. (1982). Coercive family processes (Bd. 3). Eugene: Castalia.

Pianta, R.C., Hamre, B.K., \& Allen, J.P. (2012). Teacher-student relationships and engagement: conceptualizing, measuring, and improving the capacity of classroom interactions. In S. Christenson, A. Reschly \& C. Wylie (Hrsg.), Handbook of research on student engagement (S. 365-386). New York: Springer.

Piwowar, V., \& Thiel, F. (2014). Evaluating response shift in training evaluation: comparing the retrospective pretest with an adapted measurement invariance approach in a classroom management training program. Evaluation Review, 38(5), 420-448.

Reid, J.B., Patterson, G.R., \& Snyder, J.J. (2002). antisocial behavior in children and adolescents: a developmental analysis and model for intervention. Washington, DC: American Psychological Association.

Reinecker, H. (2005). Grundlagen der Verhaltenstherapie (Bd. 3). Weinheim: Beltz PUV.

Rosseel, Y. (2012). Lavaan: An R package for structural equation modeling. Journal of Statistical Software, 48(2), 1-36. URL http://www.jstatsoft.org/v48/i02 . Zugegriffen: 20. September 2018.

Rudow, B. (2000). Der Arbeits- und Gesundheitsschutz im Lehrerberuf. Gefährdungsbeurteilung der Arbeit von Lehrerinnen und Lehrern. Heddesheim: Süddeutscher Pädagogischer Verlag.

Schaufeli, W.B., \& Taris, T.W. (2014). A critical review of the job demands-resources model: Implications for improving work and health. In G.F. Bauer \& O. Hämming (Hrsg.), Bridging occupational, organizational and public health. A transdisciplinary approach (S. 43-68). Dordrecht: Springer.

Schönbächler, M.-T. (2005). Klassenmanagement auf der Primarstufe. Dokumentation zur Datenerhebung bei den Lehrpersonen und bei den Schülerinnen und Schülern (Forschungsbericht Nr. 32). Bern: Universität Bern, Institut für Pädagogik und Schulpädagogik, Abteilung Pädagogische Psychologie. 
Schönbächler, M.-T. (2008). Klassenmanagement. Situative Gegebenheiten und personale Faktoren in Lehrpersonen- und Schülerperspektive. Bern: Haupt.

Schult, J., Münzer-Schrobildgen, M., \& Sparfeldt, J.R. (2014). Belastet, aber hochzufrieden? Zeitschrift für Gesundheitspsychologie, 22(2), 61-67.

Schwarzer, R., \& Schmitz, G.S. (1999). Lehrer-Selbstwirksamkeit. http://www.userpage.fu-berlin.de/ gesund/skalen/Lehrer-Selbstwirksamkeit/lehrer-selbstwirksamkeit.htm. Zugegriffen: 30. Dez. 2015.

Schwarzer, R., \& Warner, M. (2014). Forschung zur Selbstwirksamkeit bei Lehrerinnen und Lehrern. In E. Terhart, H. Bennewitz \& M. Rothland (Hrsg.), Handbuch der Forschung zum Lehrerberuf (2. Aufl., S. 662-678). Münster: Waxmann.

Skaalvik, E. M., \& Skaalvik, S. (2007). Dimensions of teacher self-efficacy and relations with strain factors, perceived collective teacher efficacy, and teacher burn-out. Journal of Educational Psychology, 99(3), 611-625.

Skaalvik, E.M., \& Skaalvik, S. (2017). Still motivated to teach? A study of school context variables, stress and job satisfaction among teachers in senior high school. Social Psychology of Education: An International Journal, 20(1), 15-37.

Stage, S. A., \& Quiroz, D.R. (1997). A meta-analysis of interventions to decrease disruptive classroom behavior in public education settings. School Psychology Review, 26(3), 333-386.

Tschannen-Moran, M., \& Hoy, W. A. (2001). Teacher efficacy: capturing an elusive construct. Teaching and Teacher Education, 17, 783-805.

Ulich, E., \& Wülser, M. (2004). Gesundheitsmanagement in Unternehmen. Arbeitspsychologische Perspektiven. Wiesbaden: Gabler, GWV Fachverlage.

Van de Schoot, R., Lugtig, P., \& Hox, J. (2012). A checklist for testing measurement invariance. European Journal of Developmental Psychology, 9(4), 486-492.

Van Dick, R., \& Wagner, U. (2001). Stress and strain in teaching: A structural equation approach. British Journal of Educational Psychology, 71, 243-259. 